\title{
The Centro de Investigaciones Biológicas (Center for Biological Research) in Madrid, Spain: 50 years of excellent fundamental multidisciplinary biological research
}

Nine consecutive papers published in the "Journal of Applied Biomedicine”, six of them entirely filling Volume 8, Number 3, and the last three appearing in this issue (Number 4), are short reviews on different topics, dealing with various aspects of fundamental biology and biomedicine, all nine originated in the Centro de Investigaciones Biológicas (CIB), a Spanish research institute located in Madrid, which forms part of the National Research Council of Spain (CSIC for its acronym in Spanish).

The CIB celebrated in 2009 its first 50 years of activity. Since its birth, in very difficult times in our country, CIB researchers were committed to increasing the level and international reputation of Spanish science. Over the decades, the CIB has grown and diversified, serving as the basis for the creation of new research centers; some of the most prestigious Spanish research centers in Biology, such as the Centro de Biología Molecular "Severo Ochoa”, (Center of Molecular Biology) or the Centro Nacional de Biotecnología (National Center of Biotechnology), among others, had founding members formed in CIB and coming from the CIB staff. Many university departments, throughout the whole Spanish territory, were founded or enriched by scientists whose career originated in the CIB. Nevertheless, at the same time, the continuous activity of training young people allowed the persistence of the Center as a reference institution in the international scientific community.

One of the significant features of CIB is its multidisciplinarity. The Center was founded to hold together the institutes "Ramón y Cajal" (Neurobiology), “Jaime Ferrán” (Microbiology) and “Gregorio Marañón” (Endocrinology and Metabolism). These initial specialties were increased and became more complex with the advancement of Science throughout the decades. In the meantime, the "Ramón y Cajal” Institute of Neurobiology left the
CIB and was established as a separate independent Centre. Today, with a simpler and more efficient organizational structure, research projects in CIB comprise physico-chemical studies on protein structure, molecular medicine, mechanisms of bacterial pathogenicity, immune response, cell biology and development, plant biology, pathology and biotechnology, and others. This multidisciplinary character represents a difficulty in terms of administration and management, but it offers the great advantage of facilitating a fruitful interaction between different scientific approaches.

The Editor-in-Chief of the Journal, Professor Josef Berger, has invited me to co-ordinate the publication of a series of reviews written by relevant members of the CIB in which they revised their contribution to their respective topics in the context of the recent progress of their fields with a wide perspective. I accepted with enthusiasm this task and, in turn, received a generous response from colleagues who were asked to contribute a paper.

I think that the result, in the form of nine review papers covering a relatively broad range of topics, goes beyond a mere homage to the $50^{\text {th }}$ anniversary celebration of the CIB, to offer an updated perspective of the state of art of some of the hot points of the current fundamental biology and biomedicine. I would like to express my gratitude to the authors of these papers for their work and also to the competent reviewers who have anonymously revised the manuscripts and guaranteed their scientific quality. Last but not least, thanks to the Journal's readership for their attention.

Francisco Javier Medina, Guest Editor Centro de Investigaciones Biológicas (CSIC), Ramiro de Maeztu 9, 28040 Madrid, Spain fjmedina@cib.csic.es 